\title{
MicroMundo@Salamanca (SWI). Búsqueda de nuevos microorganismos productores de antibióticos mediante una estrategia de ApS. Fomento de la actitud investigadora
}

\author{
Beatriz Santos Romero; Margarita Díaz Martínez
}

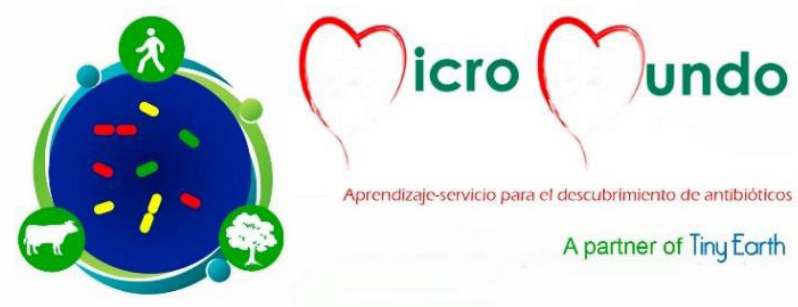

STE PROYECTO TIENE COMO OBJETIVO PRINCIPAL el fomento de las vocaciones científicas entre los alumnos de secundaria y bachillerato mediante la realización de un proyecto real de investigación por parte de los alumnos de Grado.

Para ello se han planteado los siguientes objetivos específicos:

1. Realizar un proyecto real de búsqueda de microorganismos productores de antibióticos.

2. Generar vocaciones científicas entre los alumnos universitarios y no universitarios.

3. Participación de los alumnos de Grado en foros científicos exponiendo los resultados.

El proyecto de investigación que desarrollan los alumnos de Grado se centra en la búsqueda de microorganismos productores de nuevos antibióticos a partir de muestras de suelo de diferentes hábitats según el siguiente esquema general:

1. Aislamiento de microorganismos de diferentes suelos.

2. Ensayo de antibiosis de estos aislados microbianos sobre cepas sensibles de interés.

3. Almacenamiento de las cepas de interés para el posterior estudio detallado de las moléculas producidas. 


\section{MICROMUNDO@SALAMANCA (SWI).BÚSQUEDA DE NUEVOS MICROORGANISMOS PRODUCTORES DE ANTIBIÓTICOS MEDIANTE UNA ESTRATEGIA DE APS...}

El proyecto se realiza en cinco sesiones:

1. Explicación detallada de los pasos a seguir y entrega del kit de recogida de muestras, documentos a rellenar y cuaderno de laboratorio.

2. Preparación de la muestra de suelo y siembra en placas.

3. Análisis de la diversidad de microorganismos obtenidos en los suelos y selección de 24 de ellos que serán colocados en nuevas placas.

4. Ensayo de antibiosis de los organismos seleccionados frente a una batería de microorganismos sensibles.

5. Análisis de los resultados del ensayo de antibiosis, conclusiones y preparación de las sesiones de divulgación.

En esta última sesión se realiza, además, una encuesta de satisfacción y aprovechamiento de la actividad a los estudiantes.

Los agentes implicados en el proyecto son: profesores e investigadores (SWI-IPs), alumnos de Grado de la Facultad de Biología (SWI-TAs) y alumnos y profesores de los centros de

Esta práctica profundiza en aspectos fundamentales de la carrera científica de los graduados: la docencia y la investigación.

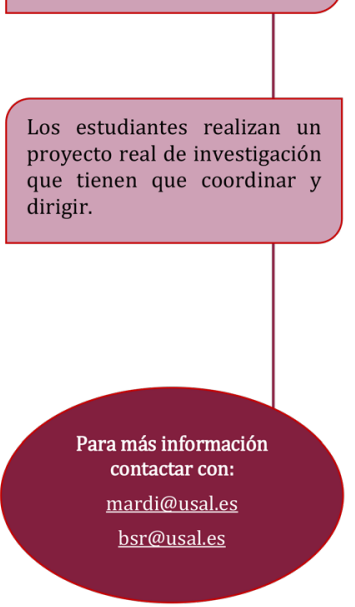
secundaria (SWI-s).

El proyecto permite utilizar material de investigación a los alumnos de secundaria al que no suelen tener acceso. Esto, junto con el trato con los alumnos de Grado, les anima a valorar la labor de los investigadores.

Con el fin de valorar la actividad se realizó un cuestionario voluntario a los alumnos de Grado participantes, cuyos resultados se pueden consultar en https://drive.google.com/ file/d/1mghX8DiYWioIy44B9TAJtHdbQ8v-h2Fm/view.

En relación con los alumnos de secundaria, la mayor parte de ellos afirma haber aumentado su interés por la investigación.

La mayor innovación en este proyecto es la aplicación de la metodología Aprendizaje y Servicio (ApS), ofreciendo a la sociedad la concienciación sobre el problema de la resistencia a los antibióticos.

Respecto a la parte investigadora, les permite darse cuenta de la dificultad y rigurosidad de la investigación real.

El proyecto supone una divulgación en sí mismo, ya que llega a los alumnos de secundaria, profesores, familias, etc. además de los ambientes universitarios. 